\title{
Driverless CBTC - specific requirements for CBTC systems to overcome operation challenges
}

\author{
M. P. Georgescu \\ Alcatel Canada Inc, Transport Automation Solutions, Train Control, \\ Canada
}

\begin{abstract}
Reliable driverless operation requires specific features implemented at system and subsystem levels of the train control system. Communications-Based Train Control (CBTC) is now proven as the best choice for driverless systems due to inherent high levels of safety and reliability with a low life cycle cost.

This paper proposes a systematic approach that may be used to determine the most efficient way to fulfil the requirements specific to each customer faced with driverless operation (green field or re-signaling). It also defines "must have" requirements (functionality) to obtain the desired performance and cost.

The paper also addresses issues related to the operability, maintainability, and availability of different types of driverless CBTC systems implementations, and the advantages and disadvantages of each solution.

Keywords: driverless, UTO, DTO, STO, CBTC.
\end{abstract}

\section{Driverless operation with Automated Train Control}

Industry members now unanimously recognize that the hierarchy for automated train control (ATC) systems is defined as Unmanned Train Operation (UTO), Driverless Train Operation (DTO), and Semi-automated Train Operation (STO) equivalent to the definitions in IEC 62290-1 (GAO2, GAO3, GAO4). For the purpose of this paper, both UTO and DTO will be expressed as Driverless operation. CBTC is defined as: Determination of train location, is to a high degree of precision, independent of Track Circuits; Continuous, bidirectional, train to wayside and wayside to train data communications, which can provide significantly more control and status information than is possible with a track 
circuit based system; Wayside and train-borne vital processors to process the train status and control data and provide continuous automatic train protection (ATP), Automatic Train Operation (ATO) and Automatic Train Supervision (ATS) functions.

Driverless systems include sophisticated central control (i.e. ATS or System Management Centres) requiring few operators as many functions are automated.

STO has a driver in the cab who is responsible for safe departure of trains from stations, while the train drives automatically between stations, often with automatic turnback functionality. The driver observes the guideway for any hazardous condition, and will stop the train if a hazardous situation is observed. Train acceleration and braking is automated and the speed is supervised continuously by the system. The driver communicates with passengers and is part of the failure recovery strategy.

With DTO, a driver is not required in the front of the train to observe the guideway for hazards. There is, however, operations staff on board. Safe departure of the train from the station, which includes train door closing, can be the responsibility of the operations staff or may be done automatically.

With UTO, operation staff is not required on board the train. Safe departure of the train from the station, including door closing, is done automatically. As well, additional systems (such as Guideway Intrusion Detection, and platform and on-board CCTV) are usually installed to support the detection and management of hazardous conditions.

\section{Driverless CBTC best fulfils train control operators' needs}

Ideally, a train control system provides (proven) safe operation, low initial capital expenditure with the best value (quality/price) for each subsystem, increased system availability/reliability, and low operating and maintenance $(\mathrm{O} \& \mathrm{M})$ costs, while providing increased operational flexibility (present and future extensions). The needs of the customer are best met by the Driverless CBTC solution.

\subsection{Driverless implementation impact on subsystems}

To implement driverless operation, design changes to several key traditional train control and auxiliary subsystems are required.

\subsubsection{Signaling}

This subsystem is crucial in providing system safety with high availability and reliability. It is also a major determinant to allow low O\&M cost; the signalling subsystem provides flexible operation, support of failure management, energy savings, low maintenance cost, and low operation cost due to reduced staff.

\subsubsection{Rolling stock}

It is critical that fault indications, particularly with regards to safety, are communicated in real time to the central control operator, not to a driver. The 
typical faults that are supervised by central (mandatory condition), are: dragging brake, motion obstructed, fire/smoke, low air pressure, park brake, slip-slide, doors status and train integrity.

Train Management System (TMS)/Health Monitoring Unit (HMU) information may be transmitted to central control and/or the maintenance shop via a separate non-vital communications link, not necessarily in real time, but still in a timely manner to trigger required unscheduled maintenance activities. It is acceptable that such information is sent via intermittent communication, e.g. downloaded from the train at each station.

"Driverless" implies automatic train control of propulsion and separate control for service, park and emergency brakes including revocable EB. Variable brake rates are implemented by the vehicle at ATC request.

Automated Speed Control is no longer a driver attribute. Sophisticated algorithms ensure jerk limitation and ride quality together with adhesion (slipslide) monitoring and compensation.

Door control without train operator involvement, door recycling, as well as reaction to 'door status lost,' will also change. This requires on-board equipment to vitally supervise indication of propulsion disabling and brake status to allow the doors to open.

Redundant on-board ATC equipment should be installed. This includes remote reset capability used to restore redundancy, in a timely manner.

Driverless operation may reduce the fleet size, due to removal of the driver's cabin, and shorter headway operation, which permit shorter trains (smaller fleet) to be used for optimum passenger service.

\subsubsection{Wayside and trackside}

2.1.3.1 Equipment. Traditional ancillary equipment is not required for true CBTC systems. This has a direct impact on capital cost, reliability/availability and O\&M cost. With true CBTC systems, 'alternate train presence detection' subsystems, based on track circuits or axle counters become an operator choice, not a requirement.

2.1.3.2 Failure management. Fault tolerant technology ensures high availability, which is obtained with (two-out-of-three) 2003 configurations that still present a cost effective solution.

ATS failure is very rare, due to redundancy and highly available components provided by today's technology. Without ATS the operator can retain fully automatic driverless operation

Wayside 'auto-restart' ensures automatic recovery following an unlikely failure of a wayside unit, to provide restoration of fully automatic operation within minutes. This sophisticated function has proven more efficient than any manual moves using "fall-back signaling" during years of reliable operation of SelTrac CBTC applied to AirTrain JFK (International Airport) and Detroit's DPM. 


\subsubsection{Depot}

Automatic operation in the depot should be considered mandatory to take full advantage of driverless operations.

Automatic routing of trains to and from storage areas, together with 'Storage Mode' for trains parked in a storage lane, and automatic 'wake-up' service to switch on necessary equipment, allows for seamless fleet build-up and reduction. This can happen any time in the operating cycle as a result of a scheduled transition, or can be triggered by Central Operator intervention as a response to an incidental change in passenger load demand. A by-product of driverless is the virtual extension of the depot in any spur and tail tracks that becomes possible, resulting in quicker changes in levels of operation.

Automatic close-up and shuffle-up in storage lanes and automatic coupling/uncoupling are powerful tools for the operator. This is important for efficiency of the operation with direct impact on energy savings and unscheduled rolling stock maintenance.

Car Wash for driverless trains travelling at low speed (e.g. $2 \mathrm{~km} / \mathrm{hr}$ ) can occur at anytime and be executed in record time.

\subsubsection{Guideway}

2.1.5.1 Infrastructure. If correlated with driverless CBTC, the civil guideway design can be optimized to achieve significant cost savings in the capital investment. Running shorter trains at lower headway has a positive impact on platform length determination.

CBTC allows for shorter end-of-guideway safety distances, which again lowers capital investment. New sections of guideway can be designed to allow significant cost savings.

Rear station turnback in conjunction with guideway and vehicle dependent safety distances results in shorter tunnels at the end of guideway.

Front station turnback design will further decrease infrastructure costs. In conjunction with FAO and an efficient alternate platform routing algorithm, the headway will remain the same as achieved by the rear turnback.

2.1.5.2 Guideway intrusion protection. Although we see them implemented in all types of train control systems, not having a driver in the cab mandates the installation of Guideway Intrusion Devices that interface with CBTC or Platform Doors subsystems.

\subsubsection{Communication Subsystems (COMMS)}

With traditional train control systems the driver provides the information and communicates with passenger in case of delays, changes of train travel, or emergencies.

For driverless operation, the communication system plays an important role and should provide the passengers with all required information, mainly in emergency situations. Due to the absence of a driver, the passengers' safety requires that the train and the wayside be equipped with a highly reliable communications system to provide on-board and platform voice information 
(e.g., next station, delays, emergency instructions in case of fire, evacuation) and emergency intercoms that allow the passengers to communicate with the central operator, on top of typical platform displays of arrival time and destination.

A CCTV subsystem becomes mandatory on the platforms and optional inside the trains.

Today's technology of voice-over IP and increased bandwidth allows for full integration of COMMS and/or CCTV functions in the ATS network.

\subsection{Operations and maintenance impact}

\subsubsection{Operating costs}

Although new jobs are created for passenger service and maintenance, CBTC facilitates lower operational costs because the drivers and their associated 'wear and tear' on the vehicle are eliminated. On top of the overall optimal system performance, the cost of driver employment, while running shorter trains, can be significant - up to about $14 \%$ of the operations cost as presented by Prof. $\mathrm{H}$. Strobel [2].

The overall operating costs are lower. Vancouver SkyTrain continues to save millions with the most efficient sized work force in the industry [1]. Direct Operating costs per passenger kilometre released by APTA 1989 [1] are presented in Figure 1.

\section{Direct Operating Cost}

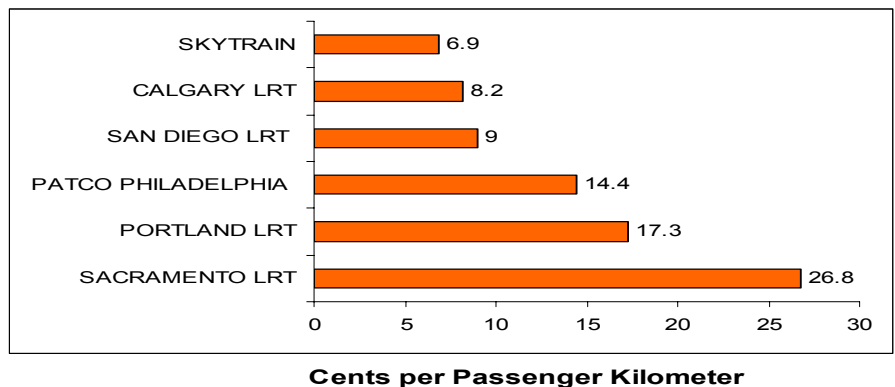

Figure 1: Operating costs per passenger kilometre.

With the cost energy increase while extending the number of stations from 16 to 32, the SkyTrain Operating cost per passenger kilometre in 2005 was 10.15 cents, way lower than Philadelphia 1986 number,

2.2.1.1 Facilitates energy optimization. With driverless operation, the optimization of regenerative braking presents the possibility of the kinetic energy of the train to be recovered during braking, by allowing train braking into a station to be coordinated with train acceleration out of another station. The regenerative energy can be consumed by the accelerating train. As stated in BC Transit's Fact Sheet - SkyTrain Performance [1], Vancouver SkyTrain "Energy consumption per passenger-kilometre is just less than half the average of the 
other systems" Figure 2 illustrates the energy consumption for North American transit industry [1] (using APTA 1989 data).

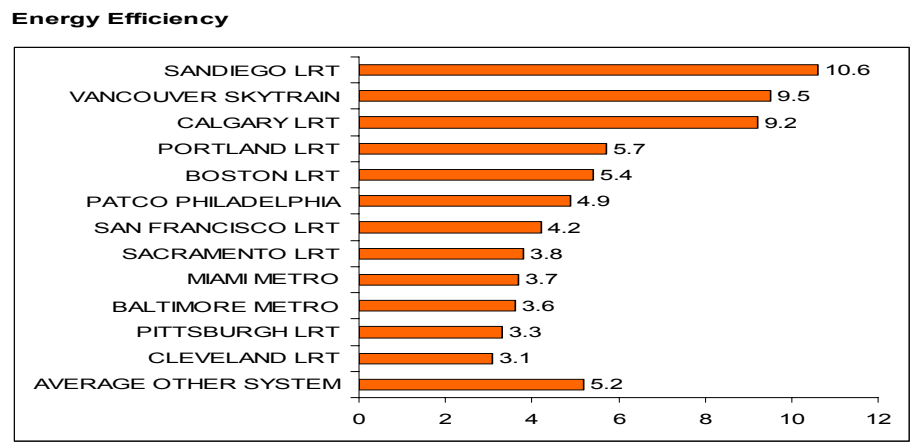

Passenger kilometres per kilowatt hour of electricity

Figure 2: $\quad$ Passenger kilometre per kilowatt hour of electricity.

In 2005, SkyTrain "Passenger Kilometre per Substation Kilowatt Hour was 6.8. This number is used to establish the traction power costs and does not include the yard, maintenance facility and stations.

2.2.1.2 Increased operational flexibility. Automated operation allows central operating staff to concentrate on any emerging problems. By design, while train performance is continuously controlled and monitored, driverless CBTC systems automatically maintain an efficient schedule. Liberated from these tasks, central control operators can concentrate on fast response to unusual situations not covered automatically by the system. Better operators' decision support is provided by intelligent CBTC systems, which can predict future conflicts long before they occur. Therefore conflicts can be avoided using the tools provided. Optimum recovery from major failures is implemented quickly. A perfect example is managing gaps in service, e.g. running supplementary unscheduled trains in parallel with scheduled trains or replacing faulty trains in service

2.2.1.3 Capacity. Firstly, capacity is increased by more frequent service of shorter trains instead of less frequent service by longer trains. Secondly, attractive options during non-peak hours are available by running variable length trains while maintaining an acceptable level of service.

\section{Driverless operation past, present, future}

The demand for driverless CBTC trend since 1981 is presented in Figure 3. The number of CBTC applications soars between 2001 and 2006 and will remain on the ascending curve. 


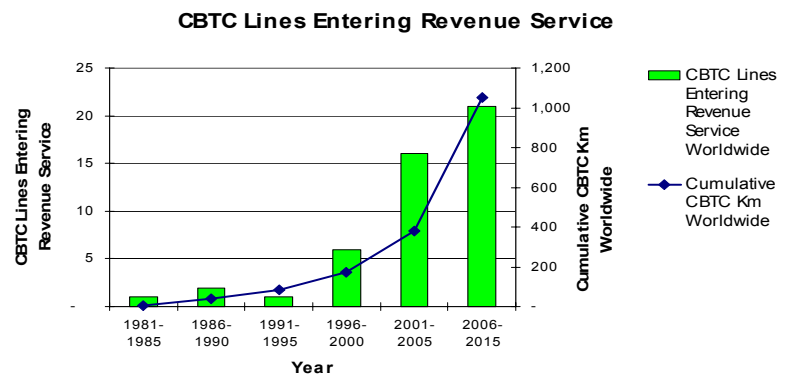

Figure 3: $\quad$ CBTC lines entering revenue service.

The split between re-signaling and green field projects presented in Figure 4 appears to indicate the transit operators' preference for the type of control (illustrated in Figure 5) at the beginning of $21^{\text {st }}$ Century. Basically most of the operators going through re-signalling with CBTC appear to prefer to keep a driver in the existing train cab, at least for the initial life of the renewed system. Green field applications options prefer UTO fully automatic operations to take advantage of the best value transit systems available as described in Moving to Full Automatic Operations [3].

CBTC Lines by New / Resignal

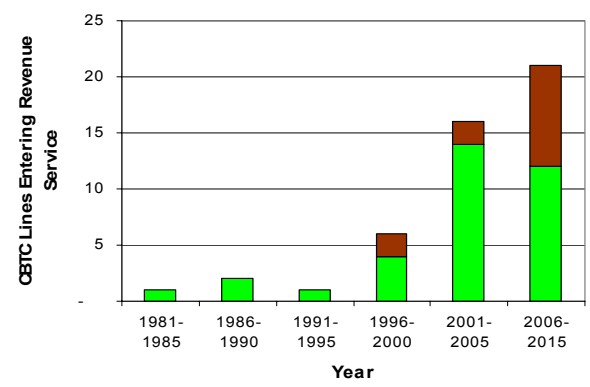

$\square$ Resignal

$\square$ New

Figure 4: $\quad$ CBTC Lines by new / resignal.

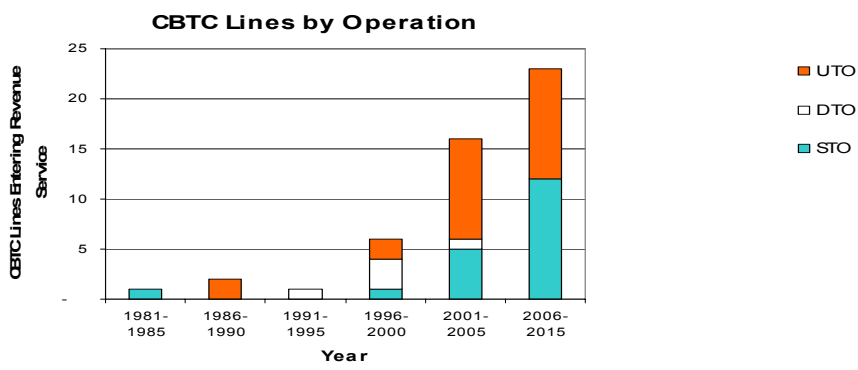

Figure 5: $\quad$ CBTC lines by operation. 
Since 1981, 46 CBTC projects have been awarded with a total of $940 \mathrm{Km}$, as illustrated in Figure 6. From this total, 58.69\% are driverless. 33.33\% of re-signaling projects selected DTO or UTO while $29 \%$ of green field projects are implementing STO.

\begin{tabular}{|c|c|c|c|c|c|c|}
\hline Customer \& Line & $\begin{array}{l}\text { Signaling } \\
\text { Company }\end{array}$ & $\begin{array}{l}\text { Type of } \\
\text { Service }\end{array}$ & Operation & $\begin{array}{c}\text { New / } \\
\text { Resignal }\end{array}$ & $\begin{array}{c}\text { Award } \\
\text { Year }\end{array}$ & $\begin{array}{c}\text { Length } \\
\mathrm{Km}\end{array}$ \\
\hline Toronto Scarborough & Alcatel & Metro & STO & New & 1981 & 6.4 \\
\hline Vancouver Sky Train & Alcatel & Metro & UTO & New & 1981 & 49.7 \\
\hline Detroit APM & Alcatel & APM & UTO & New & 1983 & 4.7 \\
\hline Lyon & Siemens & Metro & DTO & New & 1986 & 45.0 \\
\hline London DLR & Alcatel & Metro & DTO & Resignal & 1992 & 31.0 \\
\hline Paris RATP Line 14 Meteor & Siemens & Metro & UTO & New & 1992 & 7.0 \\
\hline San Francisco MUNI & Alcatel & LRT & DTO & Resignal & 1992 & 11.0 \\
\hline Ankara & Alcatel & Metro & STO & New & 1993 & 14.6 \\
\hline Kuala Lumpur & Alcatel & Metro & UTO & New & 1995 & 29.0 \\
\hline Singapore North East Line & Alstom & Metro & UTO & New & 1997 & 20.0 \\
\hline Hong Kong KCRC West Rail & Alcatel & Metro & STO & New & 1998 & 30.5 \\
\hline New York City JFK & Alcatel & Metro & UTO & New & 1998 & 13.0 \\
\hline San Francisco BART & GETS & Metro & STO & New & 1998 & 12.0 \\
\hline San Francisco Airport & Bombardier & APM & UTO & New & 1999 & 9.6 \\
\hline Seattle Airport & Bombardier & APM & UTO & New & 1999 & 2.7 \\
\hline New York City Transit Canarsie & Siemens & Metro & STO & Resignal & 1999 & 17.0 \\
\hline Las Vegas Monorail & Alcatel & APM & UTO & New & 2000 & 6.4 \\
\hline Dallas/Fort Worth Airport & Bombardier & APM & UTO & New & 2000 & 7.7 \\
\hline Philadelphia SEPTA & Bombardier & LRT & STO & Resignal & 2000 & 4.0 \\
\hline Hong Kong KCRC Ma On-Shan & Alcatel & Metro & STO & New & 2001 & 11.4 \\
\hline Nurnberg - Metro resignal & Siemens & Metro & UTO & Resignal & 2001 & 21.5 \\
\hline Singapore Marina Line & Alstom & Metro & UTO & New & 2001 & 10.6 \\
\hline Wuhan LRT & Alcatel & Metro & STO & New & 2002 & 10.2 \\
\hline Hong Kong MTRC Disney Resort Line & Alcatel & APM & UTO & New & 2002 & 3.2 \\
\hline Paris RATP Line 13 & Alcatel & Metro & STO & Resignal & 2002 & 26.0 \\
\hline Lausanne Metro & Alstom & Metro & UTO & New & 2002 & 6.0 \\
\hline Singapore Circle line Stage 3 & Alstom & Metro & UTO & New & 2002 & 19.0 \\
\hline Barcelona Line 9 & Siemens & Metro & UTO & New & 2003 & 42.0 \\
\hline Seoul KNR Bundang & Alcatel & Metro & STO & Resignal & 2003 & 18.3 \\
\hline Guangzhou Metro Line 3 & Alcatel & Metro & STO & New & 2003 & 36.0 \\
\hline Taipei Neihu Line & Bombardier & Metro & UTO & Resignal & 2003 & 15.0 \\
\hline London LUL Jubilee & Alcatel & Metro & STO & Resignal & 2003 & 35.0 \\
\hline London LUL Northern & Alcatel & Metro & STO & Resignal & 2003 & 67.0 \\
\hline Guangzhou L4L5 - Metro new line & Siemens & Metro & STO & New & 2004 & 69.0 \\
\hline Madrid Line 1\&6- Resignalling & Bombardier & Metro & STO & Resignal & 2004 & 40.2 \\
\hline Paris RATP L3 - RESIG & CSEE & Metro & STO & Resignal & 2004 & 12.0 \\
\hline Paris RATP L5 - RESIG & Siemens & Metro & STO & Resignal & 2004 & 14.5 \\
\hline London Heathrow Airport People Mover & Bombardier & APM & UTO & New & 2004 & 1.0 \\
\hline Washington Dulles Airport PM (Ph I) & Alcatel & APM & UTO & New & 2004 & 3.6 \\
\hline Yong In & Bombardier & APM & UTO & New & 2004 & 18.0 \\
\hline Shanghai Yangpu Line (M8) & Alcatel & Metro & STO & New & 2005 & 23.0 \\
\hline Budapest & Siemens & Metro & STO & Resignal & 2005 & 10.5 \\
\hline Dubai Light Metro - Red Line & Alcatel & Metro & UTO & New & 2005 & 49.0 \\
\hline Paris Line 1 & Siemens & Metro & UTO & Resignal & 2005 & 16.5 \\
\hline Beijing Airport People Mover & Bombardier & Metro & UTO & New & 2005 & 1.9 \\
\hline Vancouver Canada Line & Alcatel & Metro & UTO & New & 2006 & 18.0 \\
\hline
\end{tabular}

Figure 6: $\quad$ CBTC Projects Awards $1981-2006$.

\section{Conclusion}

With more than 20 Urban Rail Driverless Systems in revenue service worldwide and the increasing demand for driverless operation for green field and resignaling projects, the STO, DTO, UTO is an operator choice not a technology constraint.

Regardless of the solution selected, a systematic approach is required to assess customer needs. In particular, driverless operation has specific mandatory 
requirements reflected in "must have" functions to ensure reliable and efficient operation. Driverless CBTC meets today's operator challenges of increasing performance and passenger service.

\section{References}

[1] "BC Transit Fact Sheet - SkyTrain Performance", October 1991

[2] H. Strobel, J. Schuette , Presentation to UITP Conference on Automated Metros, Nurnberg 2004

[3] Firth Whitwam, Mircea Georgescu - Moving to Full Automatic Operations, IEEE Hong Kong 2005 